\title{
Camillo Golgi on Cerebellar Granule Cells
}

\author{
Paolo Mazzarello • Duane E. Haines • \\ Mario-Ubaldo Manto
}

Published online: 3 March 2012

(C) Springer Science+Business Media, LLC 2012

When Camillo Golgi made his first observations on cerebellar structure, he was working in almost complete isolation as the chief physician at the Hospital of Abbiategrasso, a small provincial town at about $23 \mathrm{~km}$ from Milan. Born in 1843 at Corteno (renamed Corteno Golgi in his honour), Golgi went on to study medicine at the University of Pavia. After his graduation in 1865 , he attended the medical practice at the Hospital of San Matteo in Pavia, where he worked with Cesare Lombroso, later a famous psychiatrist [7] and one of the founders of modern criminology, and at the Institute of General Pathology with Giulio Bizzozero, the Italian pioneer of modern cell biology [8]. After these early experiences, Golgi accepted, due to the need to find a wellpaid position, the role of chief physician at Abbiategrasso where, probably between the end of 1872 and the beginning of 1873, he developed his great methodological invention, the black reaction which, for the first time, allowed for morphological details of the brain and the spinal cord

\footnotetext{
P. Mazzarello $(\bowtie)$

Historical Museum of the University and Department

of Molecular Medicine, University of Pavia,

Pavia, Italy

e-mail: paolo.mazzarello@unipv.it

D. E. Haines

Department of Anatomy, University of Mississipi Medical center, 2500 North State Street,

Jackson, MS 39216, USA

e-mail: dhaines@umc.edu

M.-U. Manto

FNRS-Laboratoire de Neurologie Expérimentale Unité d'Etude du Mouvement (UEM), Université Libre de Bruxelles, ULB-Erasme,

Route de Lennik 808,

1070 Bruxelles, Belgium

e-mail: mmanto@ulb.ac.be
}

structure to be clearly evidenced. This important breakthrough was announced for the first time to a friend in Pavia in a letter written by Golgi on 16 February 1873 [6]:

"I spend long hours at the microscope. I am delighted that I have found a new reaction to demonstrate even to the blind the structure of the interstitial stroma of the cerebral cortex. I let the silver nitrate react on pieces of brain hardened in potassium dichromate. I have already obtained magnificent results and hope to do even better."

After only a few months, the first paper with a cursory description of the method appeared in the Italian medical journal Gazzetta Medica Italiana-Lombardia [1]. The black reaction consisted of a first phase of hardening of the tissue in potassium dichromate (for a variable period of 45 days or sometime even longer) followed by the successive and prolonged "impregnation" of the nervous elements in silver nitrate solution $(0.5-1 \%)$. The final result was the selective precipitation of a salt, silver chromate, which stained single nerve cells in black and left their prolongations nicely portrayed on a yellow background. With the application of this method, it was possible to study the morphological features of nerve cells in their morphological complexity.

Between 1873 and 1875, while still working at Abbiategrasso, Golgi carried out a series of studies using the black reaction. The first to be published was a study on the cerebellum: this paper, entitled Sulla fina anatomia del cervelletto umano ("On the fine anatomy of the human cerebellum") was first read, on 8 January 1874, at a session of the Istituto Lombardo di Scienze e Lettere (the Lombard Academy of Science and Letters) and was published, in short form, in the proceedings [3]. A longer version was subsequently published in the Archivio Italiano per le Malatie Nervose [4] and it is this version that is reproduced in this piece. 
Golgi was, by now, much more confident in the reliability of the silver chromate method. He stated that the differential staining properties of the different nervous elements was a function of the duration of the fixation in dichromate. On the basis of some modifications of the procedure, he was able to separately stain the nerve cells, the nerve fibres and the glial cells or a mixture of these elements. Golgi's paper on the cerebellum signalled his transition from the use of a "histological" level of analysis, clearly evident in the first paper reporting experiments made with the black reaction [1], to the adoption of a more "neuroanatomical" approach.

With this investigation of the cerebellar structure, Golgi demonstrated the exceptional reliability of the black reaction as a tool to penetrate the hidden, fine, structure of the central nervous system. However, the importance of this paper remained undetected for years in spite of the fact that it was summarised in some important German and English scientific journal (for reference see [6]). The results of Golgi's studies of the cerebellum, and those of his later research made with the aid of the black reaction, did not gain the international recognition they deserved until the second half of the 1880s.

Golgi remained in Abbiategrasso until the end of 1875. During this period and in addition to his work on the cerebellum, he studied the structure of the olfactory bulbs $[2,9]$ and the neuropathological alteration in a case of chorea [5]. After these studies, Golgi's scientific reputation grew, and at the end of 1875, he was appointed Professor of Histology at the University of Pavia (he became professor of General Pathology in 1879).
A new bright phase of his life then began which reached a height in 1906 when he shared a joint Nobel Prize with the Spanish neuroanatomist Santiago Ramón y Cajal. In Cerebellar Classics IX, we feature the first paper by Camillo Golgi, in which he described cerebellar granular cells, in its original format. We trust that our readers will enjoy seeing one of the original works of one of the greatest neurohistologists in history.

\section{References}

1. Golgi C. Sulla struttura della sostanza grigia del cervello (Comunicazione preventiva). Gazz Med Ital Lomb. 1873;33:244-6.

2. Golgi C. Sulla fina struttura dei bulbi olfactorii. Riv Sper Freniatr Med Leg. 1875;1:405-25.

3. Golgi C. Sulla fina anatomia del cervelletto umano. Rend Ist Lomb Sci Lett. 1874;7(serie 2):69-72.

4. Golgi C. Sulla fina anatomia del cervelletto umano. Arch Ital per le Malatie Nervose. 1874;11:90-107.

5. Golgi C. Sulle alterazioni degli organi centrali nervosi in un caso di corea gesticolatoria associata ad alienazione mentale. Riv Clin. 1874;4(serie 2):361-77.

6. Mazzarello P. Golgi. New York: Oxford University Press; 2010.

7. Mazzarello P. Cesare Lombroso: an anthropologist between evolution and degeneration. Funct Neurol. 2011;26:97-101.

8. Mazzarello P, Calligaro AL, Calligaro A. Giulio Bizzozero: a pioneer of cell biology. Nat Rev Mol Cell Biol. 2001;2:776-81.

9. Shepherd GM, Greer CA, Mazzarello P, Sassoè-Pognetto M. The first images of nerve cells: Golgi on the olfactory bulb, 1875. Brain Res Rev. 2011;66:92-105. 


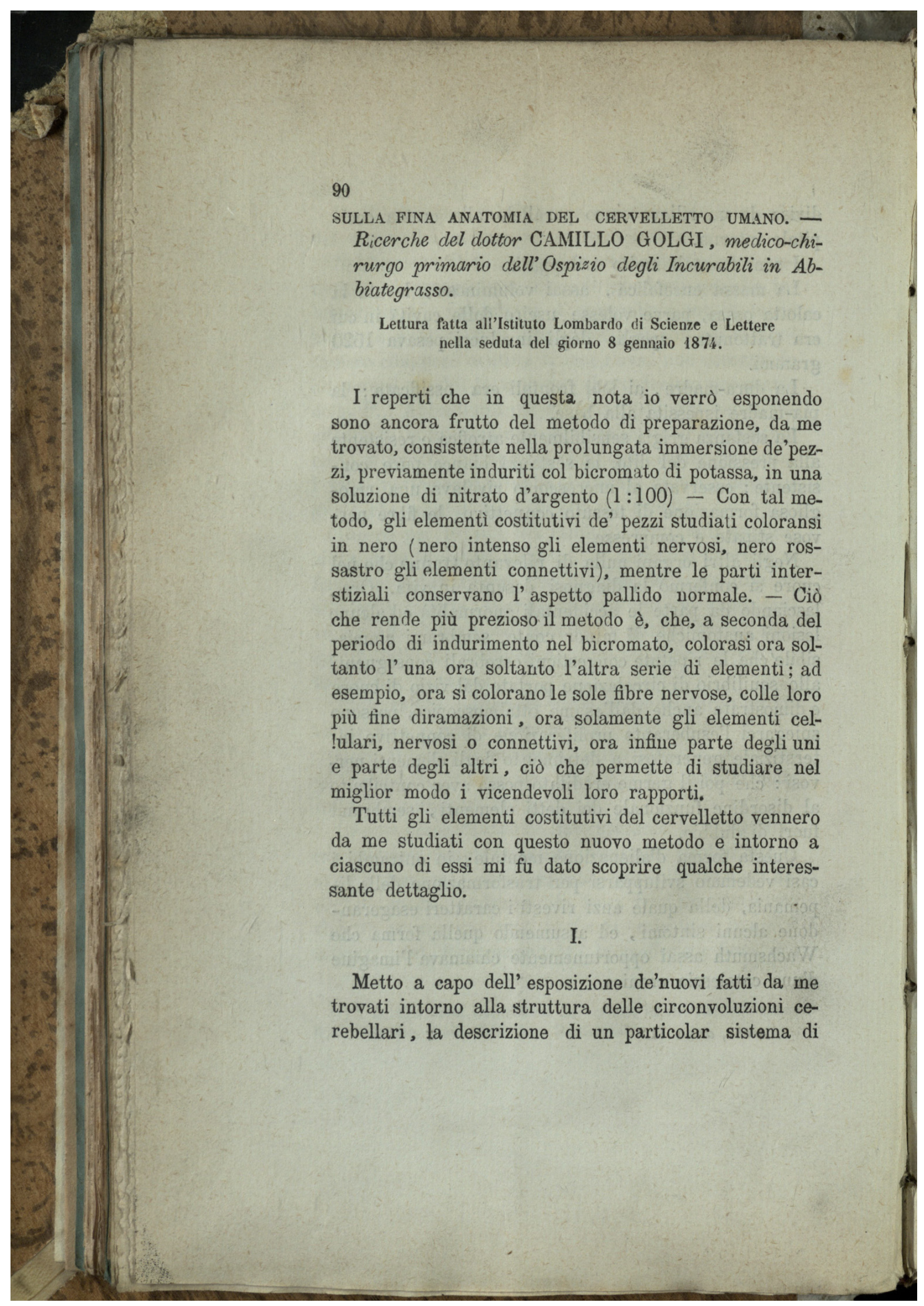


fibre nervose esistente nello strato esterno della corteccia (così detto strato molecolare). - Credo conveniente tale descrizione preliminare, sebbene, come si vedra in appresso, quel sistema di fibre si possa considerare una continuazione delle fibre che attraversano lo strato granulare, perchè alla sua formazione concorrono tutti gli elementi nervosi delle circonvoluzioni, ed altresi perchè lo studio di questo stesso sistema conduce direttamente alla soluzione de' problemi sul modo d'origine delle fibre nervose cerebellari e sulle leggi che regolano i rapporti fra le cellule e le fibre nervose. D' altra parte le fibre medesime, allorchè la reazione del nitrato d'argento si faccia nell'opportuno periodo d'indurimento (dopo un' immersione nel bicromato di 30-45 giorni nella stagione estiva, di 2-3 mesi nella stagione fredda), sembrano veramente costituire un sistema a sè.

Le fibre, sulla cui esistenza e sopra i cui rapporti intendo ora di richiamare l'attenzione dei cultori della fina anatomia, non essendo esse finora state descritte da alcun osservatore nel vero loro modo di presentarsi, trovąnsi in tutto lo strato corticale esterno del cervelletto, però sono più particolarmente spiccate, per numero e spessore, nella metà interna dello strato medesimo. In grenerale, specialmente le principali, situate nel terzo interno, decorrono fra loro parallele e sono per la massima parte di lunghezza molto considerevole, potendo molte di esse contornare perfino un'intera circonvoluzione. - Varie particolarità, oltre il lungo decorso orizzontale od arcuato, concorrono a dare a questo sistema di fibre un'impronta tutt' affatto caratteristica, cioè : 1. Le diramazioni che in tutto il loro decorso esse danno, tanto in direzione periferica, quanto in direzione centrale; 2 . Il modo di ramificarsi; i 


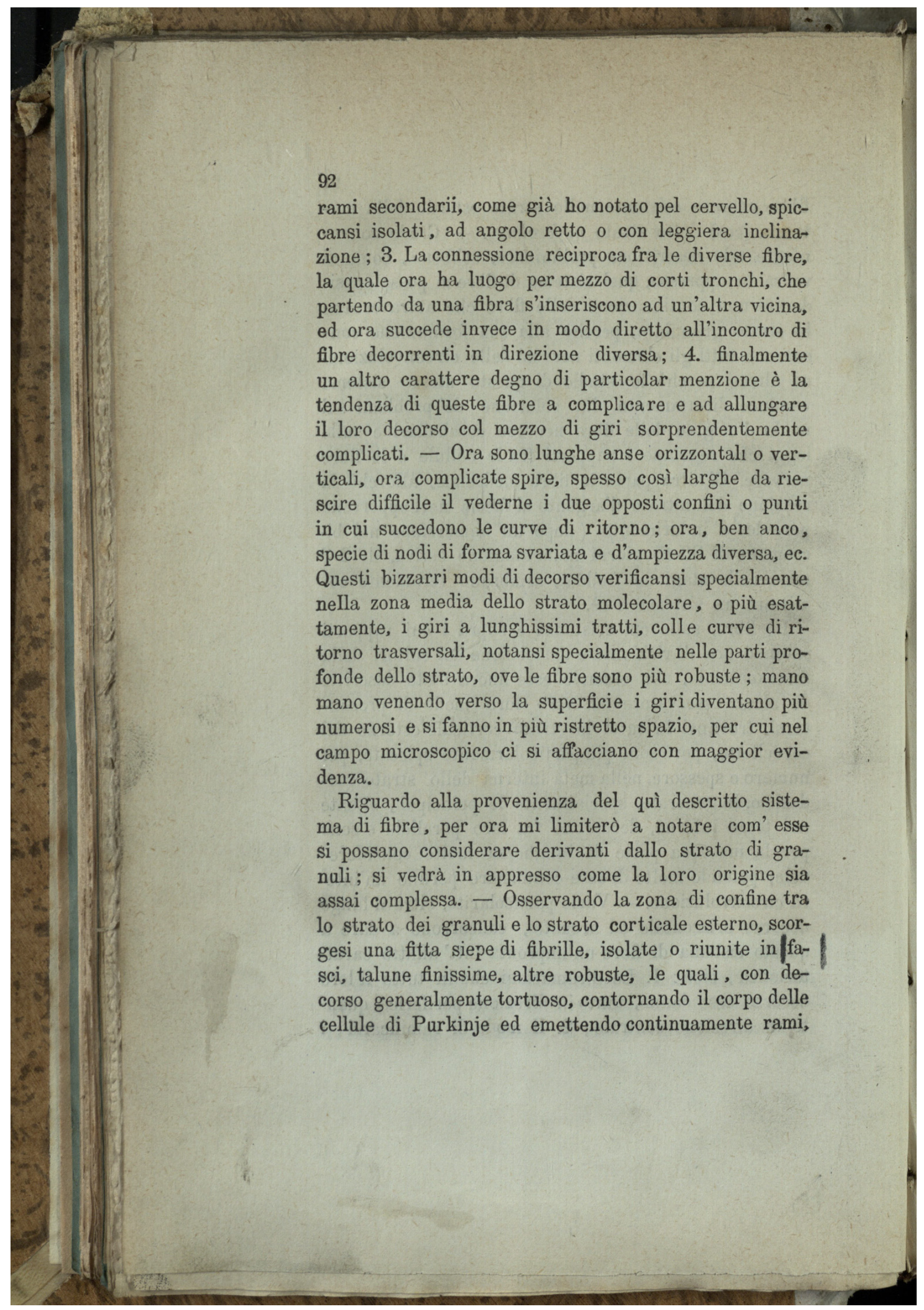


attraversano la detta zona e penetrano nello strato corticale esterno, ove subiscono vicende svariate. Alcune si inseriscono tosto (ad angolo retto, se incontrano fibre orizzontali, ad angolo acuto, con apertura in basso, se incontrano fibre ascendenti) ad altre fibre nervose che incontrano nel loro tragitto; altre ripiegansi assumendo decorso parallelo alle fibre già orizzontalmente decorrenti ; altre, prima di ripiegarsi, portansi in linea retta verso l'alto, arrivando talvolta fino verso la metà, ed anche oltre, dello strato molecolare; alcune poche fiualmente, dopo breve tragitto verticale od orizzontale, veggonsi mettere capo alle piccole cellule nervose disseminate nello strato corticale esterno; tutte poi, a distanze di una certa regolarità, continuano ad emettere filamenti, i quali, dopo un decorso più o meno lungo in direzione radiata, o s' inseriscono ad altri filamenti o ripiegansi orizzontalmente o s'incurvano in altri diversi modi, continuardo essi pure a ramificarsi.

$\mathrm{Da}$ quanto dissi sin qui si può già arguire che le fibrille formanti il complicato sistema ora descritto sono destinate a metter capo alle piccole cellule nervose dello strato molecolare. Tale modo di terminazione io riescii infatti a verificarlo in buon numero di casi ; talora l'osservai per le fibre che s'innalzano verticalmente, partendo dalle fibre arcuate; più spesso la vidi succedere soltanto dopo che quelle fibrille, da prima verticali, avevano subito complicati andirivieni ; qualche volta infine, osservai verificarsi la connessione in modo assai più diretto, per mezzo delle fibre discretamente robuste decorrenti orizzontalmente.

Da tutto questo sistema di fibre e fibrille orizzontali, oblique, verticali, tortuose, risulta un intricatissimo intreccio, senza che però si possa dire esista un 


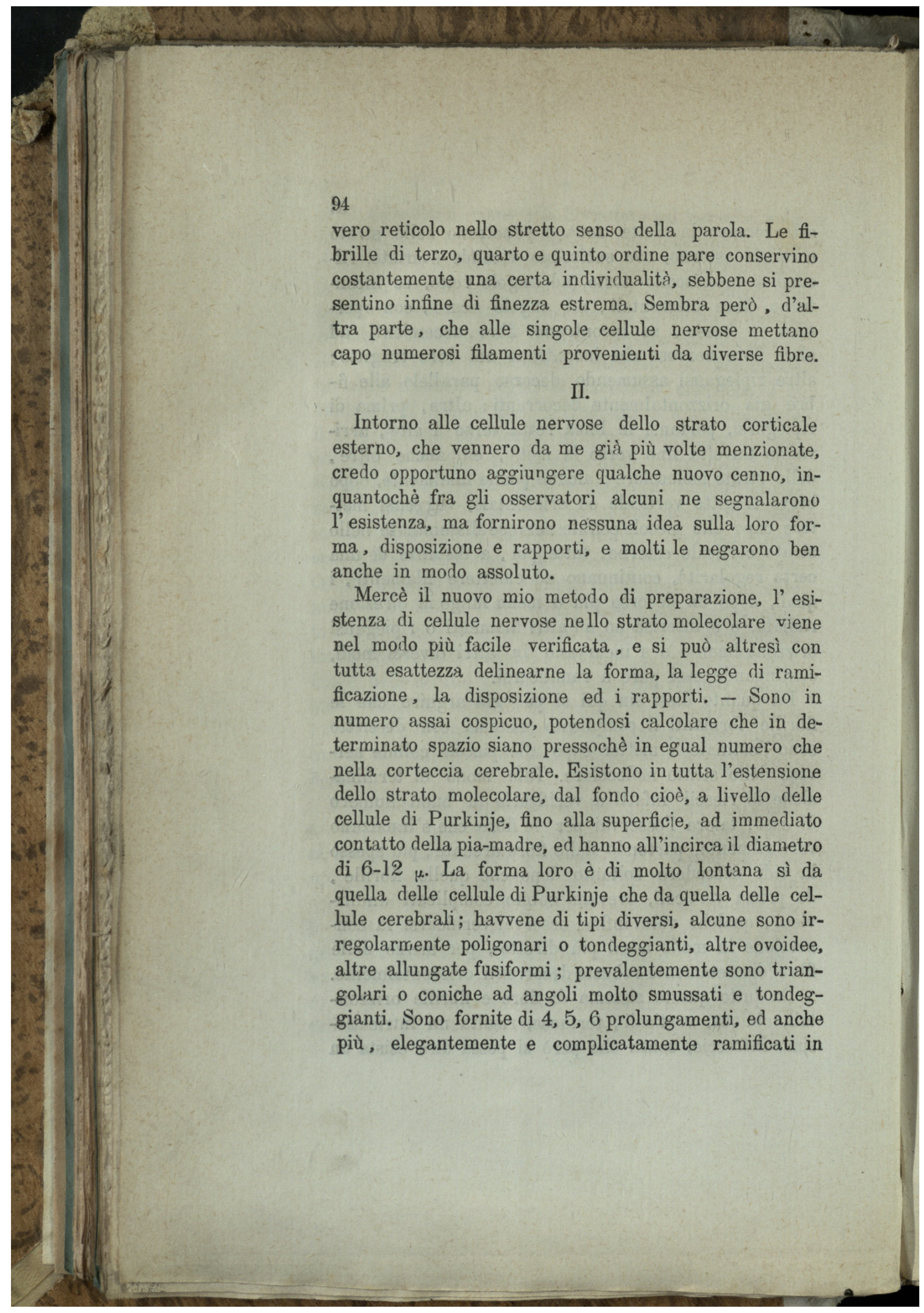


modo dicotomo, intorno alla natura dei quali vale la stessa legge che per le cellule della corteccia cerebrale; cioè uno soltanto di essi si può qualificare come essenzialmente nervoso, comechè destinato a continuarsi in una serie di fibrille nervose; agli altri conviene esattamente la denominazione di prolungamenti protoplasmatici, essendo una diretta continuazione del protaplasma cellulare e non dando origine a fibre nervose.

Questi prolungamenti, per le cellule situate verso il fondo dello strato molecolare, dirigonsi in generale verso la periferia del cervelletto, arrivando spesso fino a toccare I'estremo margine della corteccia; per le cellule situate alla periferia dello strato, la direzione dei prolungamenti protoplasmatici è, non di rado, inversa, essi portansi cioè all' ingiù verso lo strato dei granuli ; ordinariamente però in quest'ultima località, come anche più in basso, verso il mezzo dello strato, non havvi direzione ben determinata; molti dirigonsi orizzontalmente, per ripiegarsi in vario senso; alcuni vanno direttamente verso il fondo, altri verso la periferia.

Il prolungamento nervoso parte di solito da uno dei lati delle cellule per ripiegarsi poi od all'ingiù 0 verso l'alto; non di rado parte invece dalla superficie profonda; in casi non affatto rari, infine, parte dalla superficie periferica. A poca distanza dal punto d'origine $(6,10,20 \mu$.$) , questo prolungamento, dopo es-$ sersi estremamente assottigliato, comincia ad emettere filamenti per la massima parte di finezza estrema, i quali, alla lor volta, si suddividono analogamente a quanto succede nelle cellule nervose cerebrali, colla differenza che qui le ramificazioni sono molto più fine e succedono a minor distanza l'una dall'altra. - Nel 


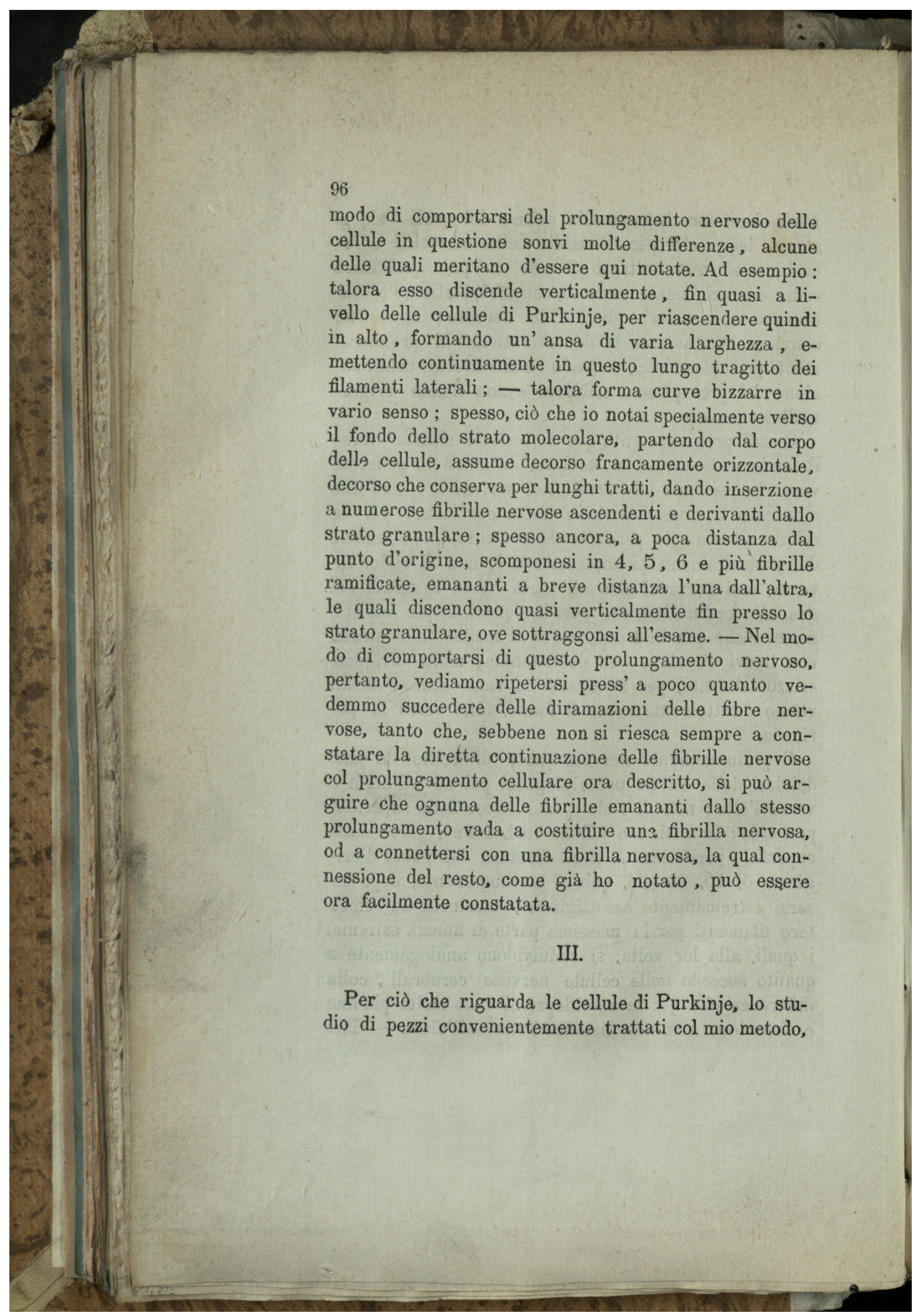


ci fa rilevare varie interessanti particolarità. Almeno il metodo del nitrato d'argento ci fa vedere, anche su sezioni, la ramificazione nei suoi più fini dettagli, al che assolutamente non poteasi riescire coi metodi sinora conosciuti.

Il modo di ramificarsi dei prolungamenti protoplasmatici ci appare notevolmente diverso da quello finora descritto dagli istologi, non escluso quello descritto in questi ultimi anni da Hadlich, Obersteiner e Boll.

Nell'insieme abbiamo le apparenze di una rete uniforme grossolana (visibile come tale coi più deboli ingrandimenti Obj. 4, oc. 3 Hartnack), continua, estendentesi dal fondo alla superficie dello strato molecolare. - Un più attento esame ci fa accorti non trattarsi di una rete in stretto senso, bensì di un intreccio fitto e complicato del quale soltanto con figure credo si possa dare un' idea prossima al vero. - Trattasi, presso a poco, di un sistema di ramificazioni emananti da tutto il contorno dei prolungamenti protoplasmatici, cominciando dalla loro prima origine dal corpo cellulare fino alle ultime suddivisioni finora descritte, le quali ramificazioni suddividendosi, incrociandosi e contorcendosi nel modo più complicato e bizzarro, riescono appunto a costituire quell'uniforme intreccio, d'apparenza reticolare, esteso dal fondo alla periferia dello strato corticale esterno.

Sul modo di terminazione delle ramificazioni dei prolungamenti protoplasmatici, non credo di potermi peranco pronunciare con sufficiente sicurezza; ritengo però di potere fin d'ora escludere ch' essi diano origine a fibre nervose, o riescano a costituire la fina rete, descritta da Boll, matrice di fibre nervose midollari.

Pì̀ interessante ancora è il modo di comportarsi 


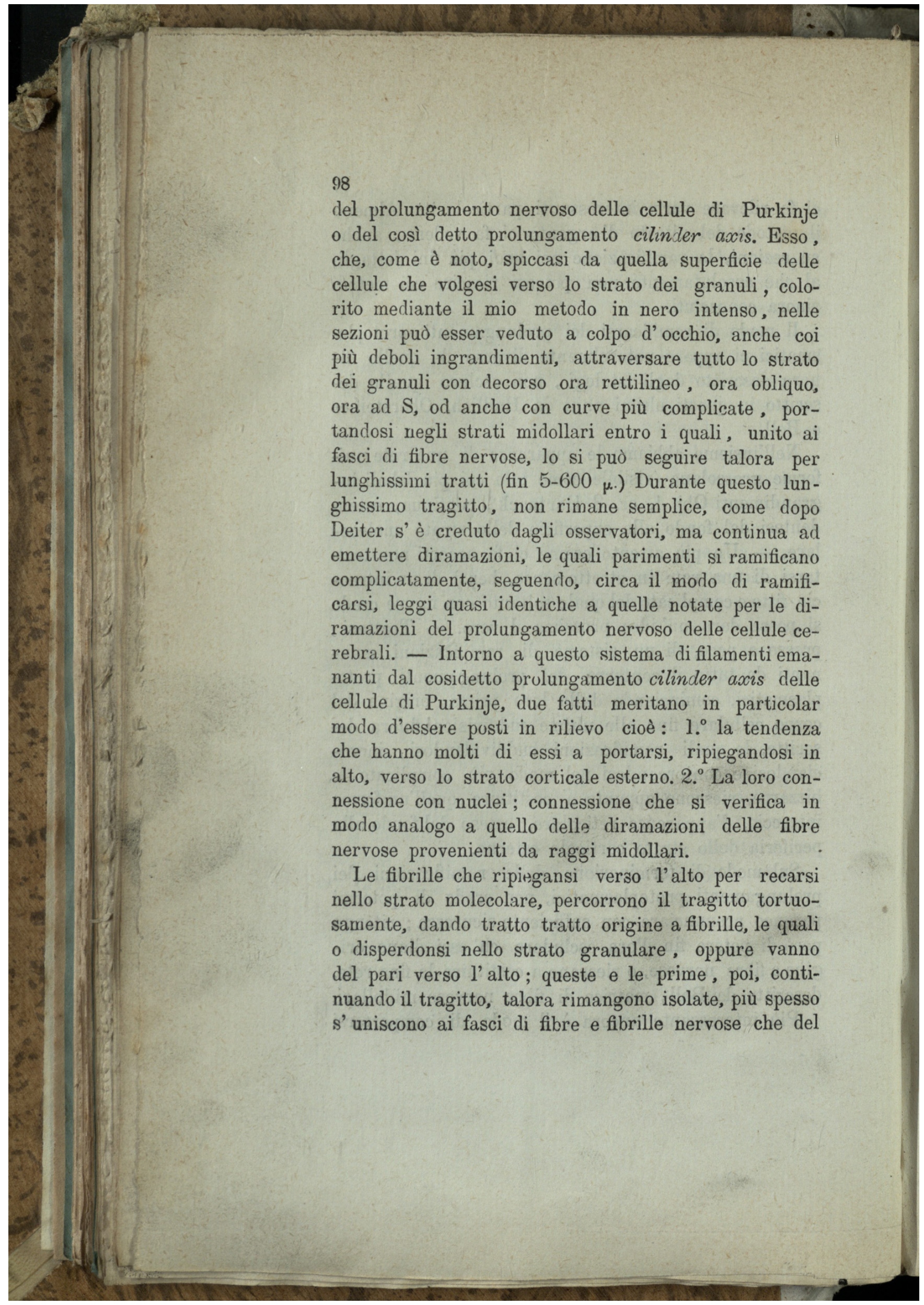


pari penetrano, nel modo suddescritto, nello strato corticale esterno. Colà pervenute di solito ripiegansi orizzontalmente, entrando a far parte del sistema di fibre dianzi descritte, colle quali anzi in alcuni casi le vidi mettersi in connessione; generalmente però, riunite e confuse colle fibre nervose, sottraggonsi all'osservazione senza che si riesca a constatare la finale loro sorte; tuttavia, argomentando dal fatto ch' esse assumono aspetto, modo di decorrere e di ramificarsi eguale a quello del sistema di fibre arcuate da me - descritto, e sopratutto argomentando dalla verificata connessione tra l'uno e l'altro sistema di fibre, parmi ovvio l' ammettere come regola generale, che le fibrille emananti dal prolungamento nervoso delle cellule di Purkinje entrino a far parte costitutiva del sistema di fibre nervose dello strato molecolare, sistema che, come vedemmo, risulta costituito, da una parte dei prolungamenti nervosi delle piccole cellule gangliari dello stesso strato molecolare, dall'altra, dai fasci di fibre provenienti dai raggi midollari, il qual sistema potrebbesi in certo modo considerare come intermediario fra le fibre midollari e le piccole cellule nervose. Ciò posto le fibrille in discorso, provenienti dalle cellule di Purkinje, sarebbero in certo modo delle fibre comunicanti tra due diverse specie di cellule.

\section{IV.}

Anche intorno alla struttura dello strato de'granuli mi fu dato scoprire alcune interessanti particolarità.

È innanzi tutto sulla natura e sui caratteri degli elementi che soglionsi comprendere sotto il nome di granuli, ch'io intendo ora pronunciarmi. 


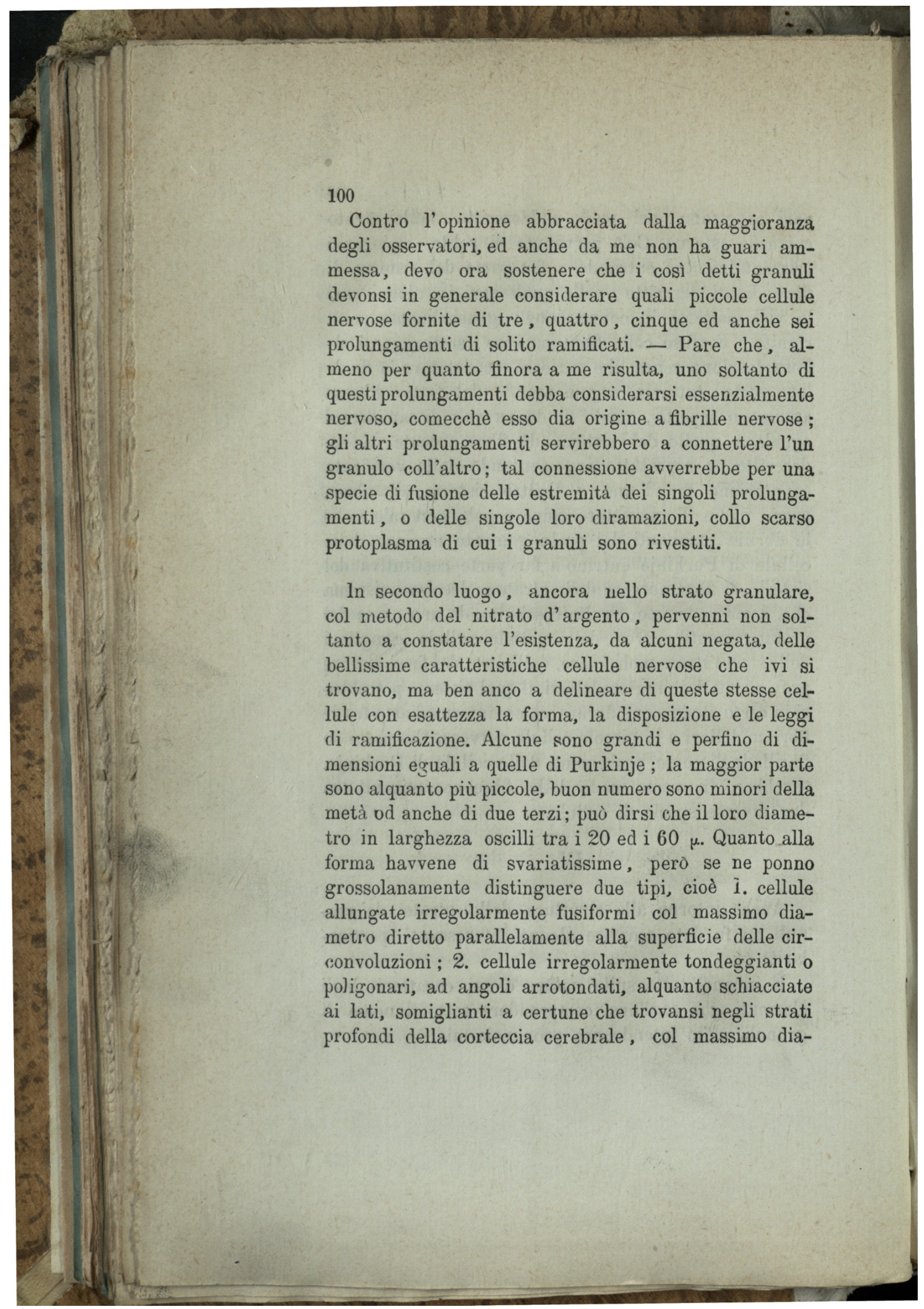


metro posto trasversalmente alla direzione dello strato granulare e perciò perpendicolare alla superficie delle circonvoluzioni. Queste e quelle sono fornite di numerosi prolungamenti, circa la natura dei quali vale sempre la legge che uno soltanto, emanante ad uno dei lati del corpo cellulare o da quella superficie di esso che volgesi verso lo strato midollare, si può considerare come essenzialmente nervoso, essendo gli altri, che ponno essere perfino in numero di sei, otto e più, di natura protoplasmatica. - Questi ultimi sono diretti in vario senso, con tendenza però ad assumere determinate direzioni, diverse pei due menzionati tipi di cellule. - Le cellule allungate inviano i loro prolungamenti protoplasmatici, alcuni dai quali di lunghezza enorme, prevalentemente verso lo strato granulare. I prolungamenti delle cellule del secondo tipo, invece, dirigonsi prevalentemente verso lo strato molecolare attraversandolo spesso in tutta la sua larghezza, ed arrivando ben anche fin proprio alla periferia. Il modo di divisione, come pei prolungamenti delle piccole cellule dello strato molecolare, è dicotomico in guisa da dar luogo ad un angolo acuto con apertura verso la periferia; e si nota che i rami secondarii, dopo un decorso divergente più o meno lungo, tendono a farsi paralleli.

Anche il prolungamento nervoso delle cellule in discorso conservasi semplice fino alla distanza di venti a trenta millimetri, quindi si ramifica complicatamente, seguendo il tipo di ramificazione proprio di tal genere di prolungamenti. - In più di un caso dal solo prolungamento nervoso d'una di queste cellule vidi, risultare per le ripetute e fine suddivisioni verificantisi in tutte le direzioni, un complicato intreccio di filamenti esteso dal fondo alla periferia dello strato 


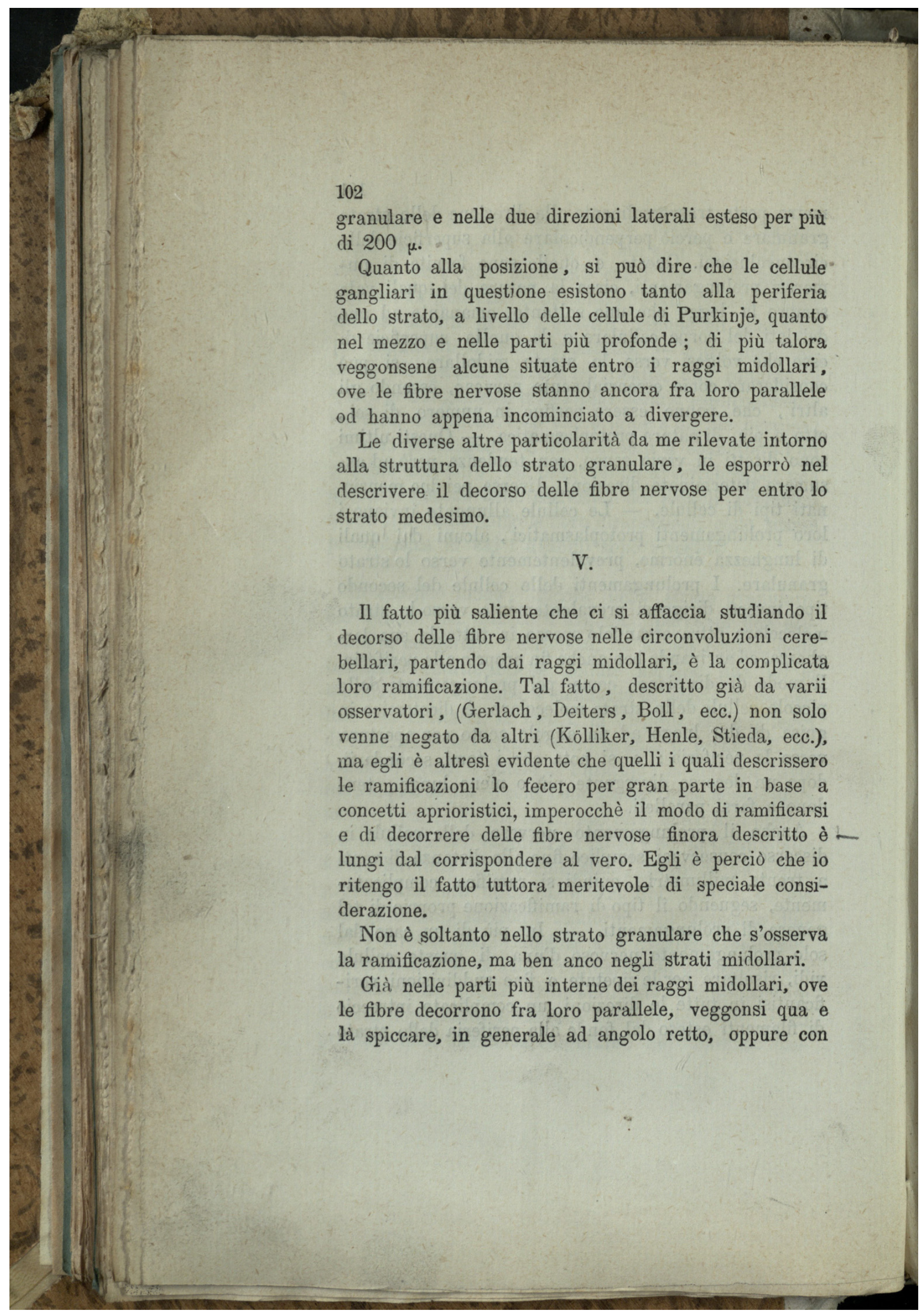


leggiera inclinazione, dalle fibre midollari che in linea retta si dirigono verso la sommità delle circonvoluzioni, delle diramazioni secondarie, ora fine, ora di diametro press' a poco eguale a quello delle fibre da cui partonó, le quali diramazioni dirigonsi verso lo strato dei granuli. In generale però le ramificazioni cominciano a farsi numerose soltanto nella zona di confine tra lo strato midollare e lo strato granulare, diventando assai frequenti in quest' ultimo strato.

Intorno al modo di comportarsi delle diramazioni delle fibre nervose nello strato de' granuli, meritano speciale rimarco le seguenti particolarità, cioè : l. la connessione coi così detti granuli e colle cellule nervose dello stesso strato granulare. 2. La connessione reciproca fra le diverse fibre. 3 . Il tortuoso, lunghissimo assai complicato decorso, analogo a quello del sistema di fibre dello strato molecolare.

La connessione delle fibre nervose coi granuli assolutamente non è quale venne in modo generale descritta da Gerlach.

Notammo come gli elementi ai quali si volle applicare la generica denominazione di granuli si possano considerare come piccole cellule nervose fornite di varii prolungamenti, dei quali, per regola generale, uno soltanto passerebbe a costituire una 0 più fibrille nervose, mentre gli altri servirebbero a connettere l' un granulo coll' altro. Se si prende ora per punto di partenza, non già i granuli, ma le fibre nervose, ci risulta che queste, nel lungo e complicato loro tragitto, inviano, specialmente dai punti nodali, numerose ramificate fibrille, le quali appunto passano a costituire il prolungamento nervoso dei singoli granuli, e sue ramificazioni; è poi ovvio il constatare come allo stesso granulo mettano capo fibrille nervose prove- 


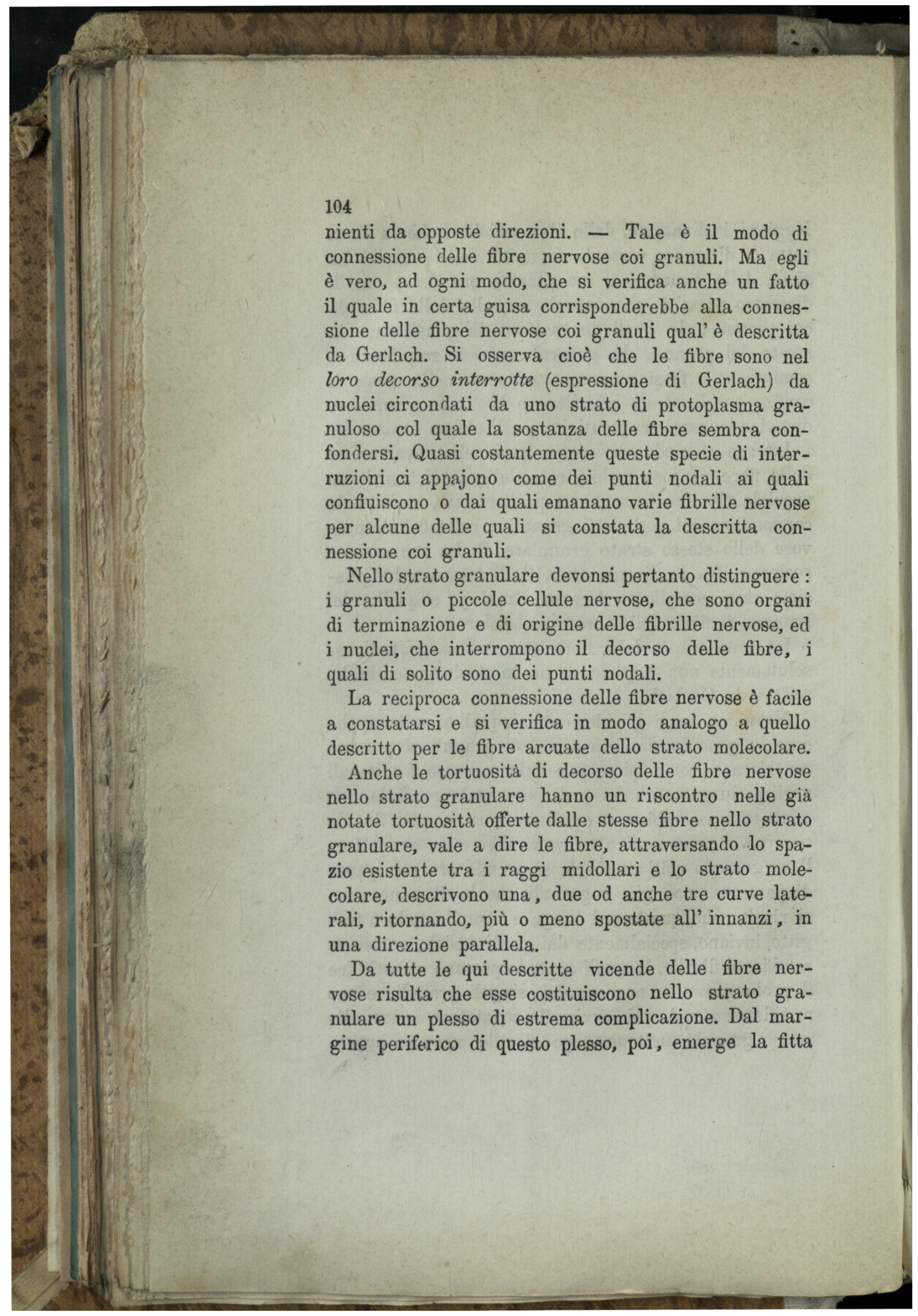


siepe di fibre o di fascetti di fibre, che, nel modo sopra descritto, unendosi alle fibre emananti dal prolungamento cilinder axis delle cellule di Purkinje, portansi a costituire il sistema di fibre arcuate dello strato corticale esterno.

Volendo ora fare un'esposizione sintetica di quanto venne da me accertato intorno al modo d'origine delle fibre nervose e intorno alle leggi che regolano i rapporti tra le cellule ele fibre nervose, parmi di poter asserire : che nel cervelletto esistono quattro sistemi di organi da cui hanno origine le fibre nervose, cioè :

1. Sistema delle piccole cellule gangliari dello strato molecolare, le quali, col loro prolungamento nervoso, in concorso dei fasci di fibre provenienti dai raggi midollari, formano il sistema di fibre nervose arcuate esistente nella metà profonda dello strato molecolare, sistema intermediario tra le fibre nervose midollari e le piccole cellule gangliari in questione.

2. Sistema delle grandi cellule di Purkinje situate nella zona di confine tra lo strato granulare ed il molecolare. Il tronco principale del prolungamento nervoso di tali cellule pare vada direttamente a costituire una fibra nervosa; i filamenti emananti dallo stesso prolungamento s'uniscono alle fibre, che, partendo dai raggi midollari, portansi allo strato molecolare per costituire il sistema di fibre arcuate dianzi accennato. In altre parole le diramazioni del prolungamento nervoso delle cellule di Purkinje sono specie di fibre comunicanti tra i due sistemi di fibre nervose provenienti dalle due specie di cellule ora accennate.

3. Sistema dei granuli formanti lo strato che da Archirio, anno $110^{\circ}$ 


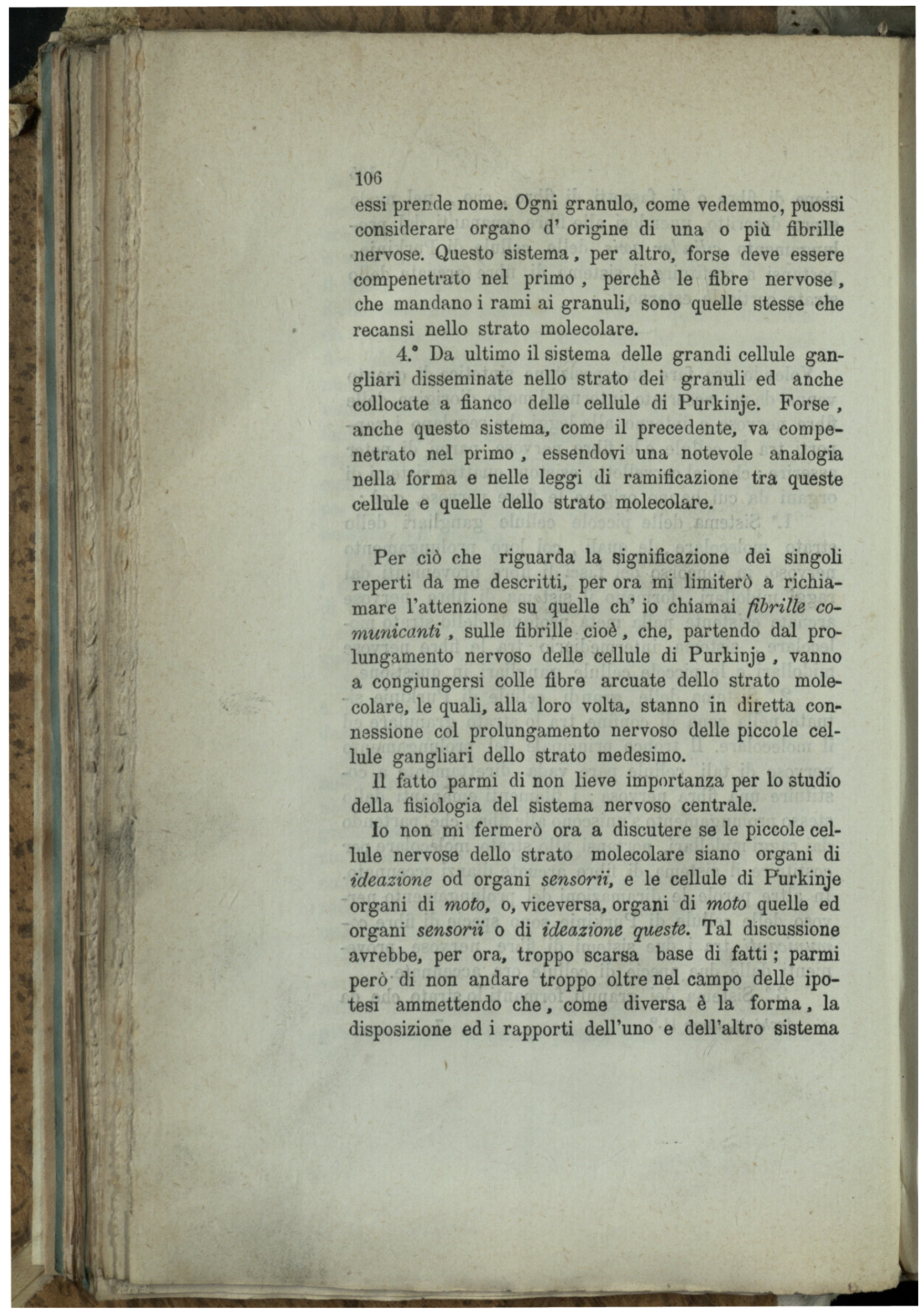


di elementi, così diverse ne siano le funzioni. - Ciò posto le fibrille in questione evidentemente assumerebbero il significato di fibre destinate a stabilire dei rapporti funzionali tra $\mathrm{i}$ due diversi sisteni di cellule.

Supponendo poi, come affatto ipoteticamente venne ammesso da alcuni, che le cellule di Purkinje siano motorie, dovrebbersi dire sensuali o psichiche le altre cellule, e in tal caso nelle fibrille comunicanti vedrebbersi rappresentate le vie per le quali l' eccitazione psichica o sensoria del primo sistema di cellule viene trasmessa o riflessa sulle cellule di Purkinje, supposte cellule motorie.

\section{RIVISTA}

Arterie nutrixie del bullo rachidiano. - I] dottor Duret divide le arterie di questo bulbo in 3 ordini.

1. Alcune sono destinate alle radici dei nervi che si staccano dal bulbo, da lui chiamate arterie radicolari;

2. Altre vanno a distribuire il sangue ai nuclei del pavimento del quarto ventricolo, verso i quali si dirigono dal solco mediano: arterie mediane o dei nuclei;

3. Altre infine si distribuiscono alle altre parti costituenti il bulbo all'avanti ed all' indietro (piramidi, olive, corpi restiformi, ecc.): arterie delle altre parti costituenti il bulbo.

Le arterie radicolari sono tutte piccole (da $1_{l^{2} 3}$ ad $1_{\not} 4$ di millimetro): procedono direttamente da un grosso tronco, (basilare, vertebrale, cerebellare): si dirigono in fuori verso le radici dei nervi, quivi si dividono in due rami, uno ascendente e l'altro discendente, dai quali dipendono i capillari che penetrano e si insinuano tra i fasci fibrillari delle radici stesse. Descrive poscia le arterie speciali che vanno alla radice di ciascun paio dei nervi cranici, ad eccezione del primo, secondo e quarto. 